\title{
Dual Arm Free Flying Space Robot Trajectory Planning Using Polynomial
}

\author{
Rishikesh Rathee and Pushparaj Mani Pathak \\ Robotics and Control Laboratory, Mechanical and Industrial Engineering Department, Indian Institute of Technology, \\ Roorkee 247667, India \\ Correspondence should be addressed to Pushparaj Mani Pathak; pushppathak@gmail.com
}

Received 22 July 2014; Revised 12 January 2015; Accepted 15 January 2015

Academic Editor: Shahram Payandeh

Copyright (C) 2015 R. Rathee and P. M. Pathak. This is an open access article distributed under the Creative Commons Attribution License, which permits unrestricted use, distribution, and reproduction in any medium, provided the original work is properly cited.

\begin{abstract}
The paper presents path planning of dual arm free flying space robot using smooth functions of time. Kinematic and dynamic modeling of dual arm free flying space robot is presented first. Using kinematic model, the Jacobian of the system has been derived, and using dynamic model, equations of motion are derived. A path planning methodology for planar system is developed using smooth function of time such as polynomials. Due to nonholonomic behaviour of the manipulator in the zero gravity environment linear and angular momentum is conserved. The proposed method yields input trajectories that drive both the manipulator and the base to a desired configuration. Joint torque curves can be obtained by introducing this joint trajectory curves in equation of motion of the space robot.
\end{abstract}

\section{Introduction}

Space introduces a complicating factor to a robotic system that is not apparent on Earth; that is, the manipulator base is not fixed in space and zero or microgravity environment exists. This introduces a high degree of dynamic complexity. Moreover, in the free flying atmosphere as the space craft actuators are turned off, the robot base has six degrees of freedom and is nonholonomic in nature. In the free flying mode, the motion of manipulator will generate reaction forces, moments on its base, and its manipulator coupling points. If no compensation is made, the robot will not attain its target since the coupling has a significant effect on the kinematics, dynamics, and control of the robot.

Path planning of free flying space robot considering nonholonomic nature of base can be done by bidirectional approach and checking its stability by defining Lyapunov function as in [1]. Yoshida et al. [2,3] have developed the equation of motion for multiple arm free flying robotic system and torque optimization for its redundant arms. They also provided an overview of its dynamics and control which was verified on ETS-VII. Papadopoulos and Moosavian [4] have used barycentric vector method for studying the dynamic behavior of multiarm space robots during chase and capture operations. Papadopoulos et al. [5] also developed a path planning methodology for single arm planar free floating space manipulator systems defining joint angles as a smooth function of polynomials.In order to overcome the difficulty that the dynamic equations of dual arm space robot system cannot be linearly parameterized, Chen and Guo [6] modelled the system as underactuated and asymptotic stability of adaptive control scheme is proved with Lyapunov method. Huang et al. [7] derive the impact dynamic equations according to the dynamic model of space robot system and proposed a genetic algorithm (GA) based on approach to search the optimal configuration of space robot at capturing moment in order to minimize or avoid the impact. Sagara and Taira [8] presented cooperative manipulation of a floating object by space robots. They also discussed application of a tracking control method using the transpose of the generalized Jacobian matrix. The dynamics control of a dual arm space robot installed on a free flying spacecraft without base position and orientation control holding a single object was discussed by Zhao et al. [9] and Siciliano and Khatib [10], and Merzouki et al. [11] presented a brief of different modelling methods and control strategies for space robots.

This paper proposes the path planning of dual arm planar space robot as a function of polynomial. It is motivated by 


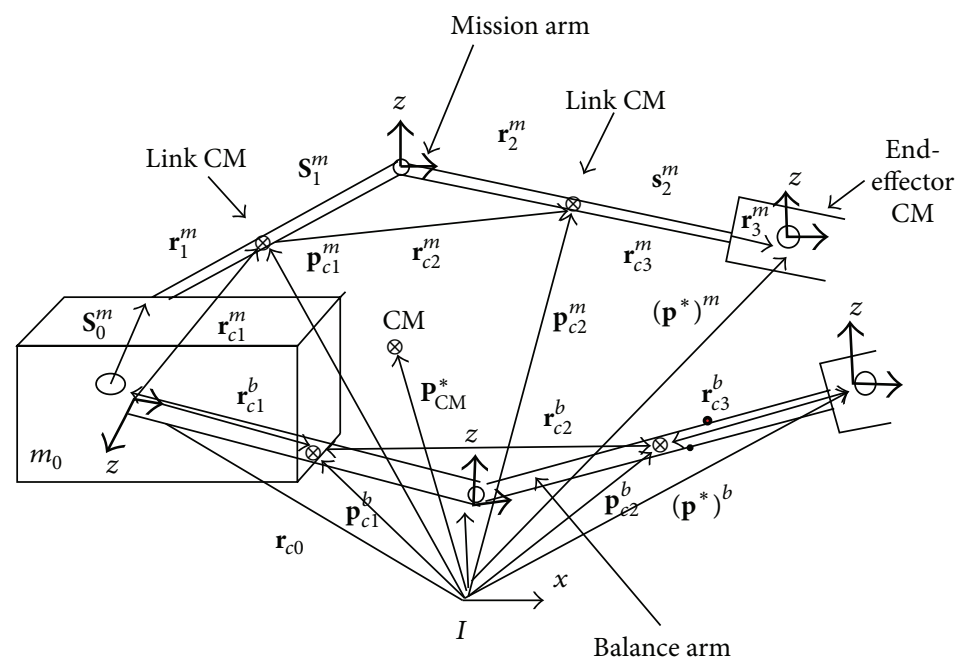

FIGURE 1: Schematic diagram of dual arm free flying space robot.

the work presented in [5]. To achieve this Jacobian [12] of dual arm free flying space robot is derived by representing its link lengths as a function of inertia parameters like mass. Equation of motion of system is derived and validated by simulation results carried out in Simulink, thus validating the model. Finally, the path planning strategy is validated by simulation results using MATLAB.

\section{Kinematic and Dynamic Modeling of Dual Arm Free Flying Space Robot}

The free flying manipulator has total $k n+6$ degree of freedom (DOF) with $n$ number of links in each $k$ number of arms where base is having six DOF [13]. Let us consider the case of dual arm $(k=2)$ free flying manipulator mounted on a base and consists of dual links $(n=2)$ in each arm as shown in Figure 1. Manipulator arms are assumed to be connected by revolute joints. Also the arms are attached on a base with the help of revolute joints. One of these arms is called mission arm $(m)$ which is used to accomplish the space mission, and the other is the balance arm $(b)$ which is used to balance the reaction motion due to the motion of the mission arm. The balance arm can also be used to accomplish the mission like the mission arm if given an appropriate trajectory to its end effector. The system center of mass (CM) remains fixed in space and the frame is considered as inertial frame. Let $\theta_{1}^{m}, \theta_{2}^{m}, \theta_{1}^{b}$, and $\theta_{2}^{b}$ be the joint angles of the joints attached to mission and balance arm. The momentum constraints are imposed due to dynamical coupling between the manipulator and the base. As we know in the absence of external forces or torque, linear and angular momentum of the system is conserved. In such a situation, the initial linear and angular momentum of the system is assumed to be zero.

The symbols required for the formulation of equations and in Figure 1 are defined as follows:

$b$ : superscript used to indicate parameter for balance arm,
Frame I: inertia frame,

Frame $i$ : $i$ th body frame or $i$ th link frame of manipulator for $i=1 \cdots n$ and for the base frame $i=0$,

$m$ : superscript used to indicate parameter for mission arm,

$m_{i}$ : mass of $i$ th body $(\mathrm{kg})$

$m_{T}$ : total mass of the system $(\mathrm{kg})$

$\mathbf{r}_{i}$ : distance from CM of $i$ th link to the preceding joint represented in the inertia frame,

$\mathbf{s}_{i}$ : distance from CM of $i$ th link to the subsequently joint represented in the inertia frame,

$\mathbf{I}_{i}$ : inertia matrix of $i$ th link with respect to inertia frame,

$\mathbf{p}^{*}$ : position vector of $\mathrm{CM}$ of the end effectors with respect to inertia frame,

$\mathbf{p}_{\mathrm{CM}}^{*}$ : position vector of $\mathrm{CM}$ of the system with respect to inertia frame,

$\mathbf{R}_{i}$ : rotation matrix of $i$ th link with respect to the base frame,

$\mathbf{J}_{n}^{*}$ : Jacobian matrix with $n$ number of links in the manipulator,

$\mathbf{M}^{*}$ : generalized inertia matrix,

$\mathrm{C}^{*}$ : centrifugal and Coriolis term,

Ø: joint variables and base variables,

$\mathbf{E}_{3}$ : identity matrix,

$\mathbf{p}_{c i}$ : position vector of $\mathrm{CM}$ of $i$ th link with respect to inertia frame,

$\mathbf{r}_{c 0}$ : position vector of $\mathrm{CM}$ of base with respect to inertia frame,

$\mathbf{r}_{c i}$ : distance between the $\mathrm{CM}$ of links, 
$\mathbf{s}_{0}$ : position vector of the first joint between the link and the base with respect to the CM of base in the inertia frame.

For free flying robotic manipulator the Denavit-Hartenberg formulation is presented in Ellery [14]. The desired end effector position for mission arm with respect to inertia frame is

$$
\begin{aligned}
\left(\mathbf{p}^{*}\right)^{m}= & \mathbf{p}_{\mathrm{CM}}^{*}+\frac{\mathbf{R}_{0}^{m} \mathbf{s}_{0}^{m} m_{0 n+1}^{m}}{m_{T}}-\frac{\mathbf{R}_{0}^{b} \mathbf{s}_{0}^{b} m_{0 n+1}^{b}}{m_{T}} \\
& +\sum_{i=1}^{n}\left(\mathbf{R}_{i}^{m} \lambda_{i}^{m}\right)-\sum_{i=1}^{n}\left(\mathbf{R}_{i}^{b} \mu_{i}^{b}\right) \\
& -\frac{\left[m_{n+1}^{m} \mathbf{R}_{n+1}^{m} \mathbf{r}_{n+1}^{m}+m_{n+1}^{b} \mathbf{R}_{n+1}^{b} \mathbf{r}_{n+1}^{b}\right]}{m_{T}},
\end{aligned}
$$

where $\lambda_{i}^{m}=\left[\left(m_{0 i}^{m}+m_{1 n+1}^{b}\right) \text { (kinematic parameter }\right)_{i}^{m}-$ $\left.m_{i}^{m} r_{i}^{m}\right] / m_{T}, \mu_{i}^{b}=\left[\left(m_{(i+1)(n+1)}^{b}\right)(\text { kinematic parameter })_{i}^{b}+\right.$ $\left.m_{i}^{b} r_{i}^{b}\right] / m_{T}$.

The desired end effector position for balance arm with respect to inertia frame is

$$
\begin{aligned}
\left(\mathbf{p}^{*}\right)^{b}= & \mathbf{p}_{\mathrm{CM}}^{*}+\frac{\mathbf{R}_{0}^{b} \mathbf{s}_{0}^{b} m_{0 n+1}^{b}}{m_{T}}-\frac{\mathbf{R}_{0}^{m} \mathbf{s}_{0}^{m} m_{0 n+1}^{m}}{m_{T}} \\
& +\sum_{i=1}^{n}\left(\mathbf{R}_{i}^{b} \lambda_{i}^{b}\right)-\sum_{i=1}^{n}\left(\mathbf{R}_{i}^{m} \mu_{i}^{m}\right) \\
& -\frac{\left[m_{n+1}^{b} \mathbf{R}_{n+1}^{b} \mathbf{r}_{n+1}^{b}+m_{n+1}^{m} \mathbf{R}_{n+1}^{m} \mathbf{r}_{n+1}^{m}\right]}{m_{T}},
\end{aligned}
$$

where $\lambda_{i}^{b}=\left[\left(m_{0 i}^{b}+m_{1 n+1}^{m}\right) \text { (kinematic parameter }\right)_{i}^{b}-$ $\left.m_{i}^{b} r_{i}^{b}\right] / m_{T}, \mu_{i}^{m}=\left[\left(m_{(i+1)(n+1)}^{m}\right)(\text { kinematic parameter })_{i}^{m}+\right.$ $\left.m_{i}^{m} r_{i}^{m}\right] / m_{T}$ :

$$
p_{\mathrm{CM}}^{*}=\frac{\left(\sum_{i=0}^{n+1} m_{i} p_{c i}^{*}\right)}{m_{T}} .
$$

Jacobian allows conversion of differential motions or velocities of individual joints to differential motions or velocities of point of interest in workspace. The free flyer Jacobian requires only the replacement of kinematic link parameters of the terrestrial manipulators with kinematic-dynamic parameters, that is, representing link lengths and joint offset as a function of mass.

Now, consider

$$
\mathbf{J}_{n}^{*}=\left[\begin{array}{l}
\mathbf{J}_{T i} \\
\mathbf{J}_{R i}
\end{array}\right]_{6 \times k n} .
$$

$\mathbf{J}_{T i}$ is $3 \times k n$ the Jacobian matrix for linear velocity of link $i$. $\mathbf{J}_{R i}$ is $3 \times k n$ the Jacobian matrix for angular velocity of link $i$.

For mission and balance arm Jacobian is

$$
\begin{aligned}
& \left(\mathbf{J}_{n}^{*}\right)^{m}=\sum_{i=1}^{n} \sum_{j=1}^{i} \frac{\partial \mathbf{R}_{i}^{m}}{\partial \theta_{j}^{m}} \lambda_{i}^{m}-\sum_{i=1}^{n} \sum_{j=1}^{i} \frac{\partial \mathbf{R}_{i}^{b}}{\partial \theta_{j}^{b}} \mu_{i}^{b} \\
& \left(\mathbf{J}_{n}^{*}\right)^{b}=\sum_{i=1}^{n} \sum_{j=1}^{i} \frac{\partial \mathbf{R}_{i}^{b}}{\partial \theta_{j}^{b}} \lambda_{i}^{b}-\sum_{i=1}^{n} \sum_{j=1}^{i} \frac{\partial \mathbf{R}_{i}^{m}}{\partial \theta_{j}^{m}} \mu_{i}^{m} .
\end{aligned}
$$

The brief concept of equation of motion of a free flying space robot as a multibody system which is presented elsewhere [7] is

$$
\mathbf{M}^{*} \ddot{\emptyset}+\mathbf{C}^{*}=\tau,
$$

where $\mathbf{M}^{*}$ is generalised inertia matrix, $\mathbf{C}^{*}$ is centrifugal and Coriolis term, $\emptyset$ is joint and base variables, and $\tau$ is external forces/moments on base and joint torques of the arm. The joint and base variable $\emptyset$ is given by

$$
\emptyset=\left[\begin{array}{llllllllll}
X_{0} & Y_{0} & Z_{0} & \theta_{0 x} & \theta_{0 y} & \theta_{0 z} & \theta_{1}^{m} & \theta_{2}^{m} & \theta_{1}^{b} & \theta_{1}^{b}
\end{array}\right]^{T},
$$

where $\left(X_{0} Y_{0} Z_{0}\right)$ are the base coordinates and $\left(\begin{array}{lll}\theta_{0 x} & \theta_{0 y} & \theta_{0 z}\end{array}\right)$ are base orientations. The generalised inertia matrix $\mathbf{M}^{*}$ is given by

$$
\mathbf{M}^{*}=\left[\begin{array}{ll}
M_{\text {Base }} & M_{\text {Coup }} \\
M_{\text {Coup }}^{T} & M_{\text {Arms }}
\end{array}\right]_{(6+k n) \times(6+k n)},
$$

where $M_{\text {Base }}=R^{6 \times 6}=$ inertia matrix of base. $M_{\text {Arms }}=R^{n \times n}=$ inertia matrix of manipulator arms. $M_{\text {Coup }}=R^{6 \times n}=$ coupling inertia matrix between base and robot manipulators. $n$ is total number of links in an arm.

These matrices can be given by

$$
\begin{aligned}
& M_{\text {base }}=\left[\begin{array}{c}
m_{T} \mathbf{E}_{3} \\
\left.m_{T} \widetilde{\mathbf{P}}_{c m} I_{0}+\left[\sum_{i=1}^{n}\left(\mathbf{I}_{i}\right)\right]^{m}+\left[\sum_{i=1}^{n}\left(\mathbf{I}_{i}\right)\right]^{b}-\sum_{i=1}^{n}\left(m_{i} \widetilde{\mathbf{P}}_{c i} \widetilde{\mathbf{P}}_{0 c i}\right)^{m}-\sum_{i=1}^{n}\left(m_{i} \widetilde{\mathbf{P}}_{c i} \widetilde{\mathbf{P}}_{0 c i}\right)^{b}\right]_{6 \times 6}
\end{array}\right. \\
& M_{\text {Arms }}=\left[\sum_{i=1}^{n}\left(\mathbf{J}_{R i}^{T} \mathbf{I}_{i} \mathbf{J}_{R i}+m_{i} \mathbf{J}_{T i}^{T} \mathbf{J}_{T i}\right)^{m}+\sum_{i=1}^{n}\left(\mathbf{J}_{R i}^{T} \mathbf{I}_{i} \mathbf{J}_{R i}+m_{i} \mathbf{J}_{T i}^{T} \mathbf{J}_{T i}\right)^{b}\right]_{k n \times k n}
\end{aligned}
$$




$$
M_{\text {Coup }}=\left[\begin{array}{c}
{\left[\sum_{i=1}^{n} m_{i} \mathbf{J}_{T i}\right]^{m}+\left[\sum_{i=1}^{n} m_{i} \mathbf{J}_{T i}\right]^{b}} \\
{\left[\sum_{i=1}^{n}\left(\mathbf{I}_{i} \mathbf{J}_{R i}+m_{i} \widetilde{\mathbf{P}}_{c i} \mathbf{J}_{T i}\right)\right]^{m}+\left[\sum_{i=1}^{n}\left(\mathbf{I}_{i} \mathbf{J}_{R i}+m_{i} \widetilde{\mathbf{P}}_{c i} \mathbf{J}_{T i}\right)\right]_{6 \times k n}^{b}}
\end{array}\right.
$$

$\mathbf{I}_{i}$ is the inertia tensor for the $i$ th link:

$$
\mathbf{I}_{i}=\left[\begin{array}{ccc}
0.5\left(-I_{x x}+I_{y y}+I_{z z}\right) & I_{x y} & I_{x z} \\
I_{x y} & 0.5\left(I_{x x}-I_{y y}+I_{z z}\right) & I_{y z} \\
I_{x z} & I_{y z} & 0.5\left(I_{x x}+I_{y y}-I_{z z}\right)
\end{array}\right]
$$

For dual arm free flying space robot,

$$
\mathbf{p}_{0 c m}=\left[\mathbf{p}_{c m}^{*}-\mathbf{r}_{c 0}\right]_{3 \times 1}, \quad \mathbf{p}_{0 c i}=\left[\mathbf{p}_{c i}-\mathbf{r}_{c 0}\right]_{3 \times 1}
$$

$\widetilde{\mathbf{P}}_{c m}, \widetilde{\mathbf{P}}_{c i}, \widetilde{\mathbf{P}}_{0 c m}$, and $\widetilde{\mathbf{P}}_{0 c i}$ are the $3 \times 3$ skew symmetric matrix of position vectors $\mathbf{p}_{c m}^{*}, \mathbf{p}_{c i}, \mathbf{p}_{0 c m}$, and $\mathbf{p}_{0 c i}$, respectively.

We have assumed that there is no external force acting on the system; therefore, the centrifugal and Coriolis term is

$$
\mathbf{C}^{*}=\dot{\mathbf{M}}^{*} \dot{\emptyset},
$$

where $\dot{\mathbf{M}}^{*}$ is the derivative of generalized inertia matrix.

\section{Path Planning for Dual Arm Free Flying Space Robot}

The angular momentum conservation cannot be integrated to analytically yield the spacecraft orientation as a function of the system's configuration. The nonintegrability property of angular momentum introduces nonholonomic characteristics to free floating systems. For simplicity we consider the planar case of the dual arm model; that is, $k n+1$ DOF system in which base is having one DOF about $z$ direction $\theta_{0}$. In this section the path planning for single arm as in [5] is implemented for dual arm case.

The linear and angular momentum conservations for both mission and balance arms are represented by the following equations:

$$
\begin{aligned}
& D_{0} \dot{\theta}_{0}+D_{1} \dot{\theta}_{1}^{m}+D_{2} \dot{\theta}_{2}^{m}=0 \\
& D_{0} \dot{\theta}_{0}+D_{3} \dot{\theta}_{1}^{b}+D_{4} \dot{\theta}_{2}^{b}=0
\end{aligned}
$$

where $D_{0}, D_{1}, D_{2}, D_{3}$, and $D_{4}$ are functions of system inertial parameters. For mission arm effect of $\theta_{1}^{b}$ and $\theta_{2}^{b}$ is neglected, hence the scleronomic constraint can be written in the form

$$
\begin{gathered}
D_{0}\left(\theta_{0}, \theta_{1}^{m}, \theta_{2}^{m}\right) d \theta_{0}+D_{1}\left(\theta_{0}, \theta_{1}^{m}, \theta_{2}^{m}\right) d \theta_{1}^{m} \\
+D_{2}\left(\theta_{0}, \theta_{1}^{m}, \theta_{2}^{m}\right) d \theta_{2}^{m}=0 .
\end{gathered}
$$

For balance arm effect of $\theta_{1}^{m}$ and $\theta_{2}^{m}$ is neglected, hence the scleronomic constraint can be written in the form

$$
\begin{gathered}
D_{0}\left(\theta_{0}, \theta_{1}^{b}, \theta_{2}^{b}\right) d \theta_{0}+D_{3}\left(\theta_{0}, \theta_{1}^{b}, \theta_{2}^{b}\right) d \theta_{1}^{b} \\
+D_{4}\left(\theta_{0}, \theta_{1}^{b}, \theta_{2}^{b}\right) d \theta_{2}^{b}=0 .
\end{gathered}
$$

The coefficients of the nonholonomic constraint become

$$
\begin{gathered}
D_{0}\left(\theta_{0}, \theta_{1}^{m}, \theta_{2}^{m}\right)=D_{0}\left(\theta_{0}, \theta_{1}^{b}, \theta_{2}^{b}\right)=\Delta_{0}, \\
D_{1}\left(\theta_{0}, \theta_{1}^{m}, \theta_{2}^{m}\right)=\Delta_{1}^{m}+\Delta_{3}^{m} \cos \left(\theta_{1}^{m}-\theta_{2}^{m}\right), \\
D_{2}\left(\theta_{0}, \theta_{1}^{m}, \theta_{2}^{m}\right)=\Delta_{2}^{m}+\Delta_{3}^{m} \cos \left(\theta_{1}^{m}-\theta_{2}^{m}\right), \\
D_{3}\left(\theta_{0}, \theta_{1}^{b}, \theta_{2}^{b}\right)=\Delta_{1}^{b}+\Delta_{3}^{b} \cos \left(\theta_{1}^{b}-\theta_{2}^{b}\right), \\
D_{4}\left(\theta_{0}, \theta_{1}^{b}, \theta_{2}^{b}\right)=\Delta_{2}^{b}+\Delta_{3}^{b} \cos \left(\theta_{1}^{b}-\theta_{2}^{b}\right),
\end{gathered}
$$

where $\Delta$ 's are the function of inertia, length, and masses of the links and base.

In general form, Inertia $=$ mass $\times(\text { radius of gyration })^{2}$

$$
\begin{gathered}
\Delta_{0}=I_{0}, \\
\Delta_{1}^{m}=I_{1}^{m}+\frac{\left[\left(r_{1}^{m}\right)^{2} m_{0} m_{1}^{m}+m_{1}^{m} m_{2}^{m}\left(s_{1}^{m}\right)^{2}+\left(a_{1}^{m}\right)^{2} m_{0} m_{2}^{m}\right]}{m_{T}}, \\
\Delta_{2}^{m}=I_{2}^{m}+\frac{\left[m_{2}^{m}\left(m_{0}+m_{1}^{m}\right)\left(r_{2}^{m}\right)^{2}\right]}{m_{T}}, \\
\Delta_{3}^{m}=\frac{\left[m_{1}^{m} m_{2}^{m} r_{1}^{m} r_{2}^{m}+m_{0} m_{2}^{m} a_{1}^{m} r_{2}^{m}\right]}{m_{T}}, \\
\Delta_{1}^{b}=I_{1}^{b}+\frac{\left[\left(r_{1}^{b}\right)^{2} m_{0} m_{1}^{b}+m_{1}^{b} m_{2}^{b}\left(s_{1}^{b}\right)^{2}+\left(a_{1}^{b}\right)^{2} m_{0} m_{2}^{b}\right]}{m_{T}},
\end{gathered}
$$




$$
\begin{aligned}
& \Delta_{2}^{b}=I_{2}^{b}+\frac{\left[m_{2}^{b}\left(m_{0}+m_{1}^{b}\right)\left(r_{2}^{b}\right)^{2}\right]}{m_{T}}, \\
& \Delta_{3}^{b}=\frac{\left[m_{1}^{b} m_{2}^{b} r_{1}^{b} r_{2}^{b}+m_{0} m_{2}^{b} a_{1}^{b} r_{2}^{b}\right]}{m_{T}} .
\end{aligned}
$$

Here $m_{0}$ and $I_{0}$ are mass and inertia of base and $m_{1}^{m}, m_{2}^{m}, m_{1}^{b}$, $m_{2}^{b}$ and $I_{1}^{m}, I_{2}^{m}, I_{1}^{b}, I_{2}^{b}$ are masses and inertia of links 1 and 2 of mission and balance arm, respectively. $m_{T}$ is total mass of the system. $a$ is the link lengths of respective links, $l$ is the distance from $j-1$ joint to center of mass of that link, $r$ is the distance from center of mass to $j$ th joint. Note that $a=l+r$ for every link.

Therefore, from the above equations we get

$$
\begin{aligned}
I_{0} d \theta_{0} & +\left(\Delta_{1}^{m}+\Delta_{3}^{m} \cos \left(\theta_{1}^{m}-\theta_{2}^{m}\right)\right) d \theta_{1}^{m} \\
& +\left(\Delta_{2}^{m}+\Delta_{3}^{m} \cos \left(\theta_{1}^{m}-\theta_{2}^{m}\right)\right) d \theta_{2}^{m}=0 \\
I_{0} d \theta_{0} & +\left(\Delta_{1}^{b}+\Delta_{3}^{b} \cos \left(\theta_{1}^{b}-\theta_{2}^{b}\right)\right) d \theta_{1}^{b} \\
& +\left(\Delta_{2}^{b}+\Delta_{3}^{b} \cos \left(\theta_{1}^{b}-\theta_{2}^{b}\right)\right) d \theta_{2}^{b}=0 .
\end{aligned}
$$

Equation (18) contains three differentials. Path planning can be done if this form is transformed to the equations consisting of two differentials. So nonintegrable equations of the form of (18) can be written as

$$
\begin{aligned}
& d u+v \cdot d w=0 \\
& d x+y \cdot d z=0
\end{aligned}
$$

where $u, v$, and $w$ are properly selected functions of $\theta_{0}, \theta_{1}^{m}$, and $\theta_{2}^{m}$ and $x, y$, and $z$ are properly selected functions of $\theta_{0}$, $\theta_{1}^{b}$, and $\theta_{2}^{b}$.

For mission arm and balance arm the forward transformation is given by

$$
\begin{gathered}
u\left(\theta_{0}, \theta_{1}^{m}, \theta_{2}^{m}\right)=\Delta_{0} \cdot \theta_{0}+\Delta_{2}^{m} \cdot \theta_{2}^{m}-\Delta_{3}^{m} \cdot \sin \left(\theta_{1}^{m}-\theta_{2}^{m}\right) \\
v\left(\theta_{0}, \theta_{1}^{m}, \theta_{2}^{m}\right)=\Delta_{1}^{m}+2 \Delta_{3}^{m} \cdot \cos \left(\theta_{1}^{m}-\theta_{2}^{m}\right) \\
w\left(\theta_{0}, \theta_{1}^{m}, \theta_{2}^{m}\right)=\theta_{1}^{m} \\
x\left(\theta_{0}, \theta_{1}^{b}, \theta_{2}^{b}\right)=\Delta_{0} \cdot \theta_{0}+\Delta_{2}^{b} \cdot \theta_{2}^{b}-\Delta_{3}^{b} \cdot \sin \left(\theta_{1}^{b}-\theta_{2}^{b}\right) \\
y\left(\theta_{0}, \theta_{1}^{b}, \theta_{2}^{b}\right)=\Delta_{1}^{b}+2 \Delta_{3}^{b} \cdot \cos \left(\theta_{1}^{b}-\theta_{2}^{b}\right) \\
z\left(\theta_{0}, \theta_{1}^{b}, \theta_{2}^{b}\right)=\theta_{1}^{b}
\end{gathered}
$$

These equations constitute a transformation $\left(\theta_{0}, \theta_{1}^{m}\right.$, $\left.\theta_{2}^{m}\right) \rightarrow(u, v, w)$ for mission arm and $\left(\theta_{0}, \theta_{1}^{b}, \theta_{2}^{b}\right) \rightarrow(x, y, z)$ for balance arm which is defined at every point of the configuration space of the system. Moreover, the orientation $\theta_{1}^{m}$ and $\theta_{1}^{b}$ are conserved through the transformation and are explicitly available for planning; that is, one can directly set a desired trajectory for $\theta_{1}^{m}$ and $\theta_{1}^{b}$.
Therefore, the planning problem reduces to choosing functions $f$ and $g$ given by (22). We choose function $f$ as a fifth-order polynomial and $g$ can be forth-order polynomial, so that while finding coefficients of them the system initial and final configuration, velocity, and acceleration can be satisfied:

$$
\begin{gathered}
w=z=f(t)=c_{5} t^{5}+c_{4} t^{4}+c_{3} t^{3}+c_{2} t^{2}+c_{1} t+c_{0}, \\
u=x=g(w)=b_{4} w^{4}+b_{3} w^{3}+b_{2} w^{2}+b_{1} w+b_{0}, \\
v=y=-g^{\prime}(w)=-\left(4 b_{4} w^{3}+3 b_{3} w^{2}+2 b_{2} w+b_{1}\right) .
\end{gathered}
$$

Here the coefficients ( $c$ 's) of polynomial $w$ are computed using the initial and final values of orientation $\theta_{1}^{m}$, angular velocity $\dot{\theta}_{1}^{m}$, and angular acceleration $\ddot{\theta}_{1}^{m}$. The coefficients of polynomial $z$ are computed using the initial and final values of orientation $\theta_{1}^{b}$, angular velocity $\dot{\theta}_{1}^{b}$, and angular acceleration $\ddot{\theta}_{1}^{b}$. But for mission and balance arm the inverse transform is defined if and only if the constraints given by (23) are satisfied:

$$
\begin{aligned}
& -1 \leq\left(\frac{v-\Delta_{1}^{m}}{2 \Delta_{3}^{m}}\right) \leq 1, \\
& -1 \leq\left(\frac{y-\Delta_{1}^{b}}{2 \Delta_{3}^{b}}\right) \leq 1 .
\end{aligned}
$$

These constraints have been implied from $\theta_{2}^{m}$ and $\theta_{2}^{b}$ values derived from (24) and (25). For mission arm one uses initial and final conditions of $\theta_{0}, \theta_{1}^{m}$, and $\theta_{2}^{m}$ in (20) in order to find $u_{\text {int }}, u_{\text {fin }}, v_{\text {int }}$, and $v_{\text {fin }}$ and using the polynomial Equation (22) we get unknown coefficients $b_{3}, b_{2}, b_{1}$, and $b_{0}$ for mission arm. For balance arm one uses initial and final conditions of $\theta_{0}, \theta_{1}^{b}$, and $\theta_{2}^{b}$ in (21) to find $x_{\text {int }}, x_{\text {fin }}, y_{\text {int }}$, and $y_{\text {fin }}$ and using the polynomial equation (22) we get unknown coefficients $b_{3}$, $b_{2}, b_{1}$, and $b_{0}$ for balance arm:

$$
\begin{gathered}
\theta_{0}=\frac{1}{\Delta_{0}}\left[u-\Delta_{2}^{m} w-\Delta_{2}^{m} \cos ^{-1}\left(\frac{v-\Delta_{1}^{m}}{2 \Delta_{3}^{m}}\right)\right. \\
\left.-\Delta_{3}^{m} \sqrt{1-\left(\frac{v-\Delta_{1}^{m}}{2 \Delta_{3}^{m}}\right)^{2}}\right], \\
\theta_{1}^{m}=w, \\
\theta_{2}^{m}=w+\cos ^{-1}\left(\frac{v-\Delta_{1}^{m}}{2 \Delta_{3}^{m}}\right) .
\end{gathered}
$$

Similarly, for balance arm we have

$$
\begin{gathered}
\theta_{0}=\frac{1}{\Delta_{0}}\left[x-\Delta_{2}^{b} z-\Delta_{2}^{b} \cos ^{-1}\left(\frac{y-\Delta_{1}^{b}}{2 \Delta_{3}^{b}}\right)\right. \\
\left.-\Delta_{3}^{b} \sqrt{1-\left(\frac{y-\Delta_{1}^{b}}{2 \Delta_{3}^{b}}\right)^{2}}\right]
\end{gathered}
$$


TABLE 1: Parameters used in simulation of DC motor.

\begin{tabular}{lc}
\hline Moment of inertia of rotors $(J)$ & $0.01 \mathrm{kgm}^{2}$ \\
Damping ratio of motors shafts $(b)$ & $0.0001 \mathrm{Nms}$ \\
Electromotive force constant $\left(K_{t}=K_{e}=K\right)$ & $0.01 \mathrm{Nm} / \mathrm{Amp}$ \\
Electric resistance of the two motors $(R)$ & $0.1 \mathrm{ohm}$ \\
Electric inductance of motors $(L)$ & $0.5 \mathrm{H}$ \\
\hline
\end{tabular}

TABLE 2: Parameters used in simulating of space robot.

\begin{tabular}{lc}
\hline Mass of base $\left(m_{0}\right)$ & $4 \mathrm{~kg}$ \\
Mass of first link of mission and balance arm $m_{1}^{m}=m_{1}^{b}$ & $0.2942 \mathrm{~kg}$ \\
Mass of second link of mission and balance arm including masses of their grippers $m_{2}^{m}=m_{2}^{b}$ & $0.2942 \mathrm{~kg}$ \\
Length of first link of mission and balance arm $a_{1}^{m}=a_{1}^{b}$ & $0.4 \mathrm{~m}$ \\
Length of second link of mission and balance arm $a_{2}^{m}=a_{2}^{b}$ & $0.3 \mathrm{~m}$ \\
Distance of CM of base to first joint of both the arms $\left(s_{0}^{m}\right)=\left(s_{0}^{b}\right)$ & $0.5 \mathrm{~m}$ \\
Inertia of first link of mission and balance arm $I_{1}^{m}=I_{1}^{b}$ & $0.03 \mathrm{~kg} \mathrm{~m}^{2}$ \\
Inertia of second link of mission and balance arm including end effectors inertia $I_{2}^{m}=I_{2}^{b}$ & $0.02 \mathrm{~kg} \mathrm{~m}^{2}$ \\
Inertia of base $\left(I_{0}\right)$ & $0.4 \mathrm{~kg} \mathrm{~m}^{2}$ \\
\hline
\end{tabular}

$$
\begin{gathered}
\theta_{1}^{b}=z, \\
\theta_{2}^{b}=z+\cos ^{-1}\left(\frac{y-\Delta_{1}^{b}}{2 \Delta_{3}^{b}}\right) .
\end{gathered}
$$

The polynomial $v$ is written as a function of $b_{4}$ and the unknown spacecraft final orientation $\theta_{0}^{\text {fin }}$. So $b_{4}$ and $\theta_{0}^{\text {fin }}$ are chosen in such a way that it satisfies constraint in (23). Once $f$ and $g$ are found, the trajectories or $\theta_{0}, \theta_{1}^{m}, \theta_{2}^{m}, \theta_{1}^{b}, \theta_{2}^{b}$ are found using (24) and (25), which shows the inverse transformation from $u, v$, and $w$ to $\theta_{0}, \theta_{1}^{m}, \theta_{2}^{m}$ and from $x, y$, and $z$ to $\theta_{0}, \theta_{1}^{b}$, $\theta_{2}^{b}$. It is seen that both the arms have a cumulative effect on orientation of base $\theta_{0}$.

\section{Simulation and Results}

Dynamic model derived in (6) of space robot is simulated using MATLAB and Simulink software. Simulation of equation of motion is obtained by providing step input to four DC motors located at the four joints of the dual arm robot. Modelling of DC motor is obtained using the following equations:

$$
\begin{gathered}
J \frac{d^{2} \theta}{d t^{2}}=T-b \frac{d \theta}{d t}, \\
L \frac{d i}{d t}=-R i+V-e, \\
T=K_{t} i, \\
e=K_{e} \frac{d \theta}{d t} .
\end{gathered}
$$

In the above equation (26), $J$ is moment of inertia of rotor, $\theta$ is motor rotation, $T$ is motor torque, $b$ is damping ratio,
$L$ is electric inductance of motors, $i$ is armature current, $R$ is armature resistance, $V$ is voltage supplied, $e$ is back electromotive force (emf), and $K_{t}$ and $K_{e}$ are electromotive force constants. Table 1 shows the parameters used in simulation of DC motor. Table 2 shows the parameters used for simulation of space robot.

End effectors trajectory is obtained by first simulating joint motions of the robot and then transferring it to its tip using Jacobian given by (5). To validate equations of motion of the proposed model, base disturbance caused due to the effect of mass and inertia of links should be negligible, hence increasing base mass to $400 \mathrm{~kg}$ and inertia to $300 \mathrm{~kg} \mathrm{~m}^{2}$. The two links of each arm are made straight and joint between them is locked by increasing the motor damping $B_{2}$ by 100 times. As expected the two end effectors of mission arm and balance arm plot a circular trajectory as shown in Figure 2, with radius equal to the summation of their dynamic link lengths.

For simulation of path planning parameters used are same as given in Table 2 and the duration of motion is chosen equal to $10 \mathrm{~s}$. The free-floater has to move its manipulator endpoint to a new location and at the same time change its spacecraft attitude to a desired one. Only manipulator actuators are to be used. Let initial system configuration of mission arm be $\left(\theta_{1}^{m}, \theta_{2}^{m}\right)^{\text {in }} \equiv\left(0^{\circ}, 30^{\circ}\right)$ and the final be $\left(\theta_{1}^{m}, \theta_{2}^{m}\right)^{\text {fin }} \equiv\left(30^{\circ}, 60^{\circ}\right)$. Let initial system configuration of balance arm be $\left(\theta_{1}^{b}, \theta_{2}^{b}\right)^{\text {in }} \equiv$ $\left(180^{\circ}, 150^{\circ}\right)$ and the final be $\left(\theta_{1}^{b}, \theta_{2}^{b}\right)^{\text {fin }} \equiv\left(140^{\circ}, 100^{\circ}\right)$. The initial system configuration of base is $\left(\theta_{0}\right)^{\text {in }} \equiv 0^{\circ}$ and the final is $\left(\theta_{0}\right)^{\text {fin }} \equiv 5^{\circ}$.

This requirement may result in a range of possible $b_{4}$. Of these, $b_{4}$ is chosen so that the range of allowable final spacecraft attitudes is maximized. For this case, for both the arms $b_{4}=20$. Joint torques curves can be obtained by simplifying (6) to the planar case. 


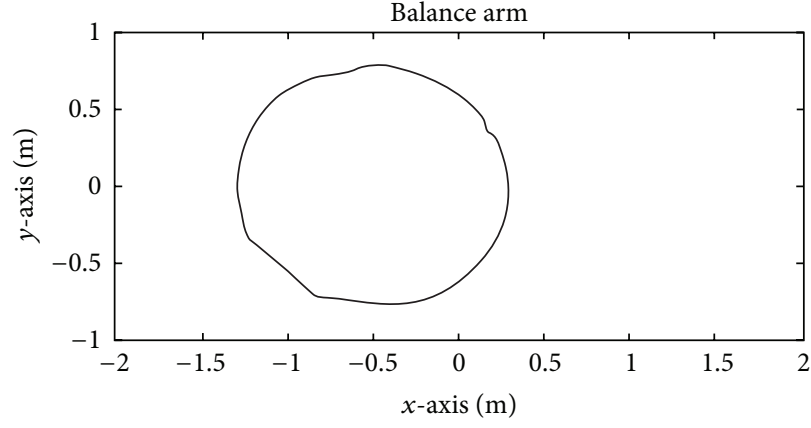

(a)

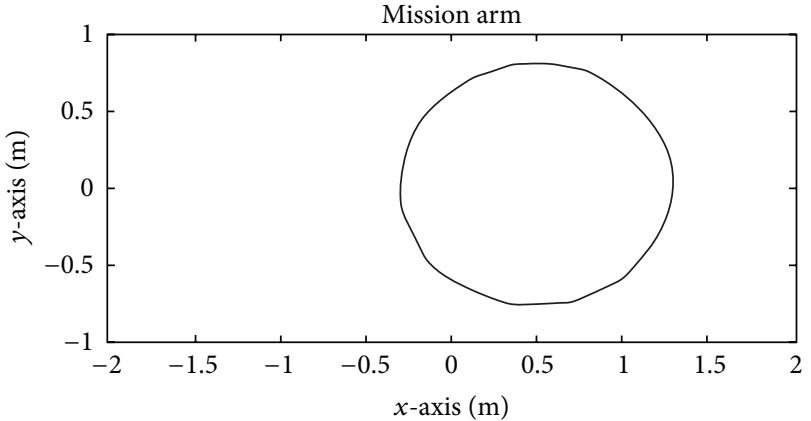

(b)

FIgURE 2: Plot of $X_{\text {tip }}$ v/s $Y_{\text {tip }}$ of dual arm space robot for both the arms.

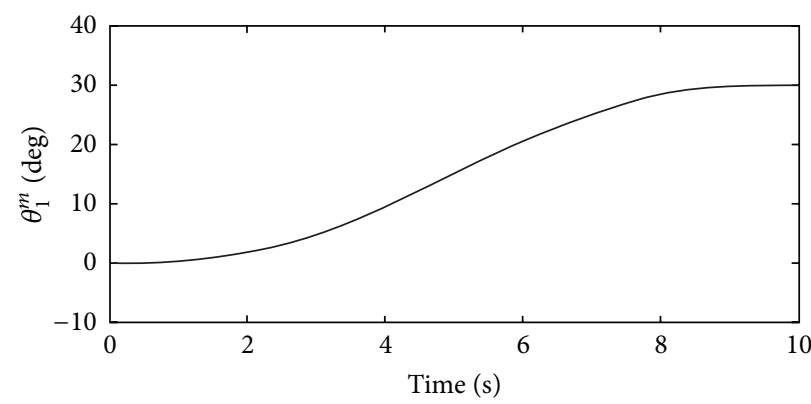

(a)

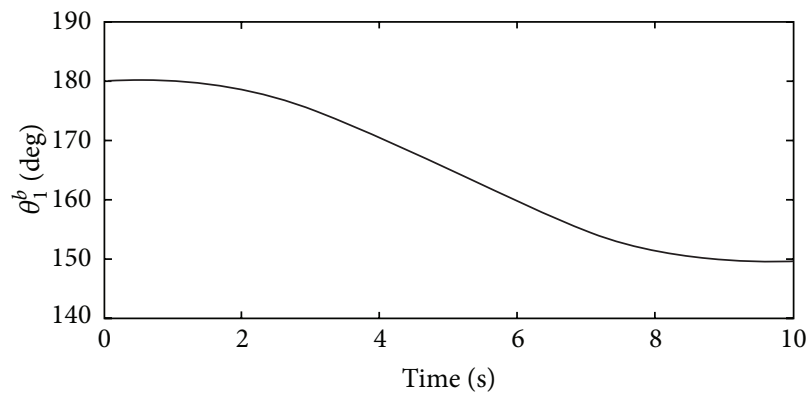

(c)

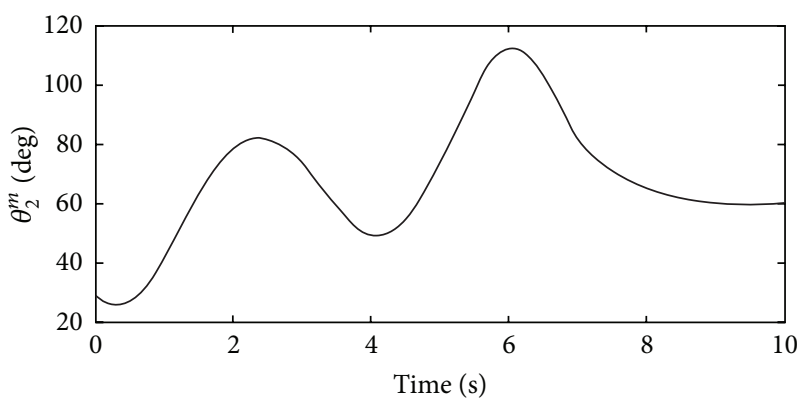

(b)

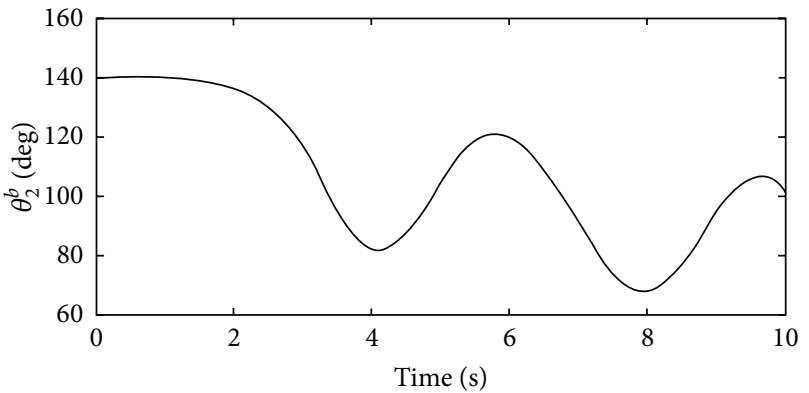

(d)

FIGURE 3: Path followed by all four joints of both the arms using polynomial approach for $\left(\theta_{1}^{m}, \theta_{2}^{m}\right)^{\text {in }} \equiv\left(0^{\circ}, 30^{\circ}\right),\left(\theta_{1}^{m}, \theta_{2}^{m}\right)^{\text {fin }} \equiv\left(30^{\circ}, 60^{\circ}\right)$, $\left(\theta_{1}^{b}, \theta_{2}^{b}\right)^{\text {in }} \equiv\left(180^{\circ}, 150^{\circ}\right)$, and $\left(\theta_{1}^{b}, \theta_{2}^{b}\right)^{\text {fin }} \equiv\left(140^{\circ}, 100^{\circ}\right)$.

As shown in Figure 3, the desired configuration is reached in the specified time. Also, all trajectories are smooth throughout the motion, and the system starts and stops smoothly at zero velocities, as expected and shown in Figures 4,5 , and 6 . This is an important characteristic of the method employed and is due to the use of smooth functions, such as polynomials. As shown in Figure 7, the required torques can be easily applied by the joint actuators to reach the final configuration. From Figure 8, total base orientation changes from $53.27^{\circ}$ to $-6.85^{\circ}$ which contains the desired $5^{\circ}$, but the total base disturbance is around $60^{\circ}$ which is undesirable. Increasing or decreasing simulation time and base parameters keeping in mind the singularities has no effect on the path taken but increases or decreases the torque requirements and the magnitude of velocities or accelerations. Hence, increasing the base mass to $10 \mathrm{~kg}$ and its inertia to $1 \mathrm{~kg}-\mathrm{m}^{2}$ for feasible solution we can minimize total base disturbance to $10^{\circ}$ as shown in Figure 9.

\section{Conclusions}

In this paper dynamic modeling and path planning of dual arm free flying space robot are presented. Equation of motion for the case of dual arm free flying is derived and simulated in Simulink. Results show that if the base mass and inertia are increased to a certain limit keeping in mind 


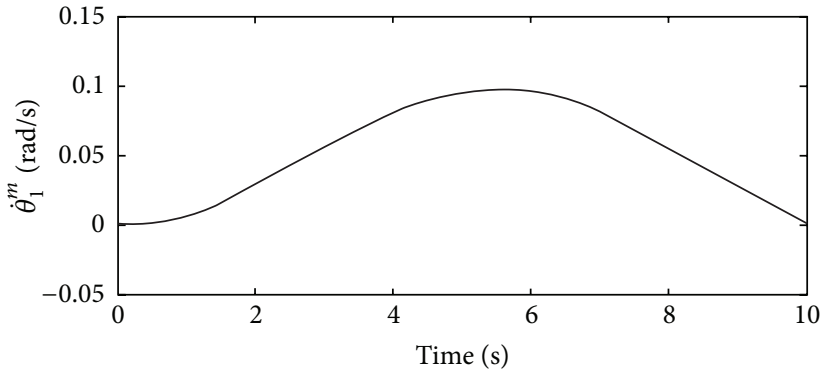

(a)

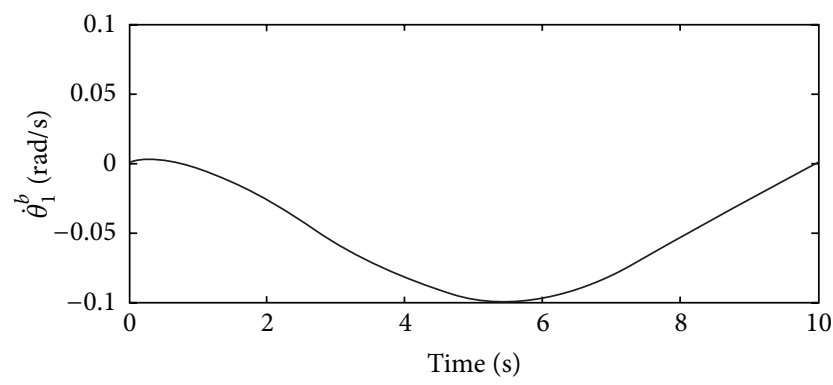

(c)

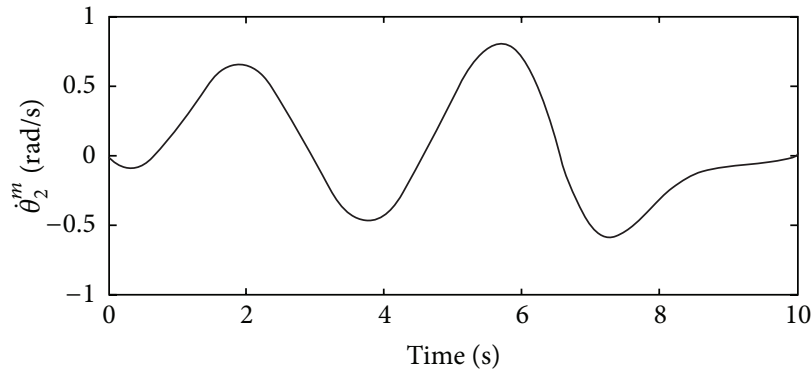

(b)

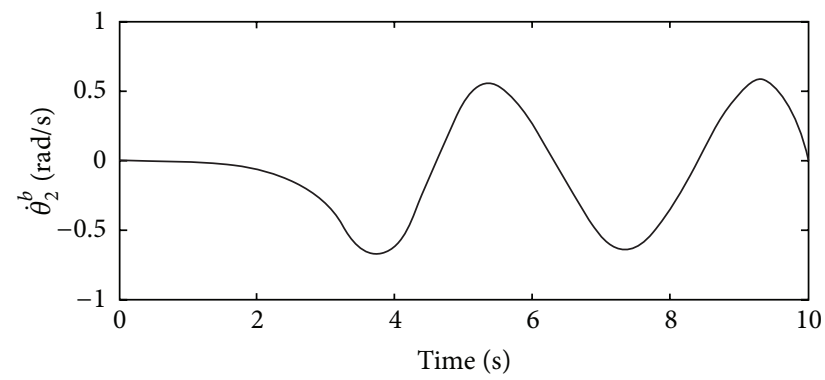

(d)

FIGURE 4: Rate of change of joint angles of both the arms.

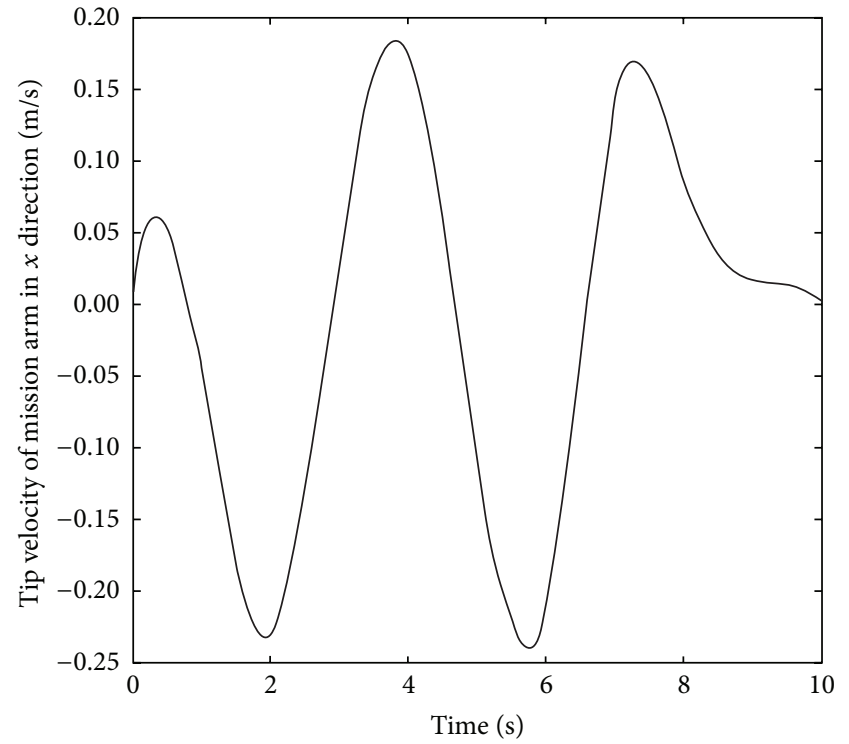

(a)

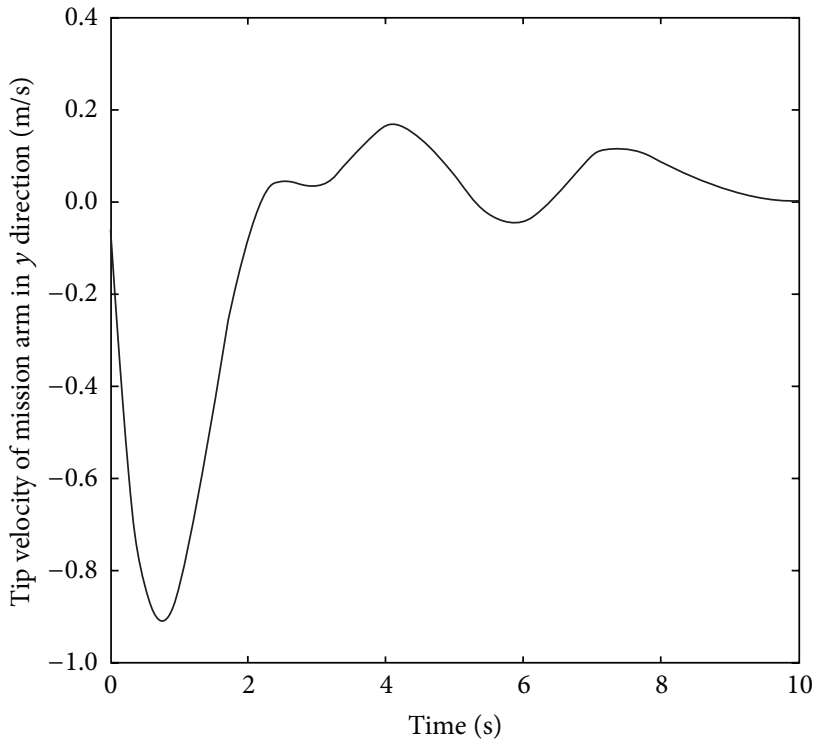

(b)

FIGURE 5: End effectors velocity of mission arm.

the singularities, then base disturbance can be decreased and the end effectors follow the required circular path. A path planning methodology was implemented for dual arm free flying space manipulators using smooth and continuous functions such as polynomials. The desired configuration is reached in the specified time. Also, all joint trajectories are smooth throughout the motion, and the system starts and stops smoothly at zero velocities, as expected. This is an important feature of the method used and is due to the use of functions, such as polynomials. The required torques is small and torque variation is smooth and can be easily be applied by the joint actuators to reach the final configuration. 


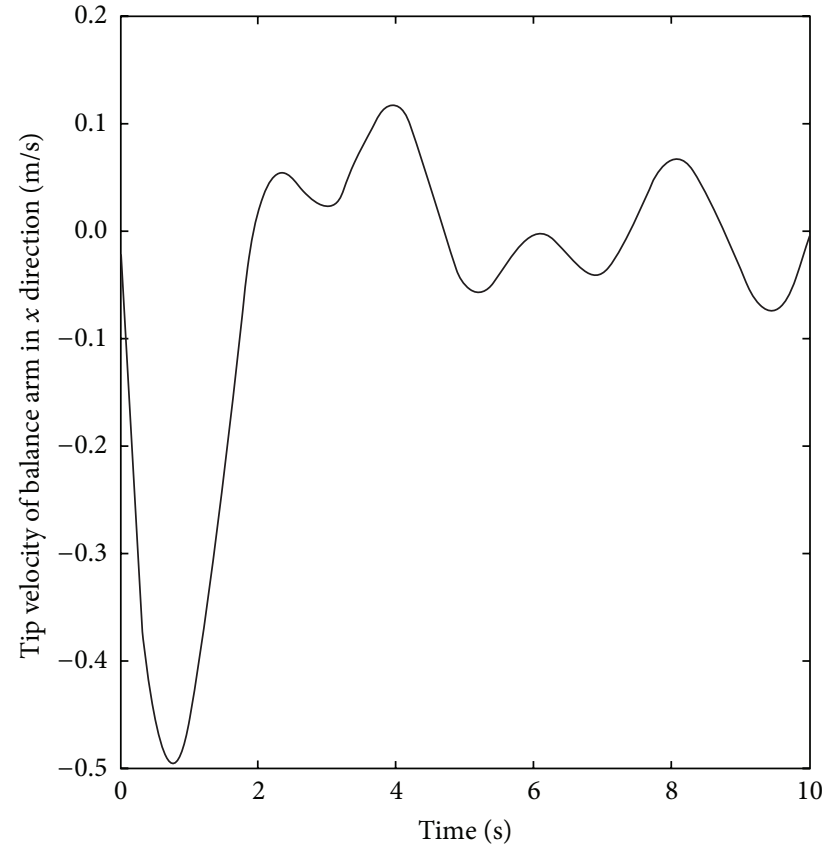

(a)

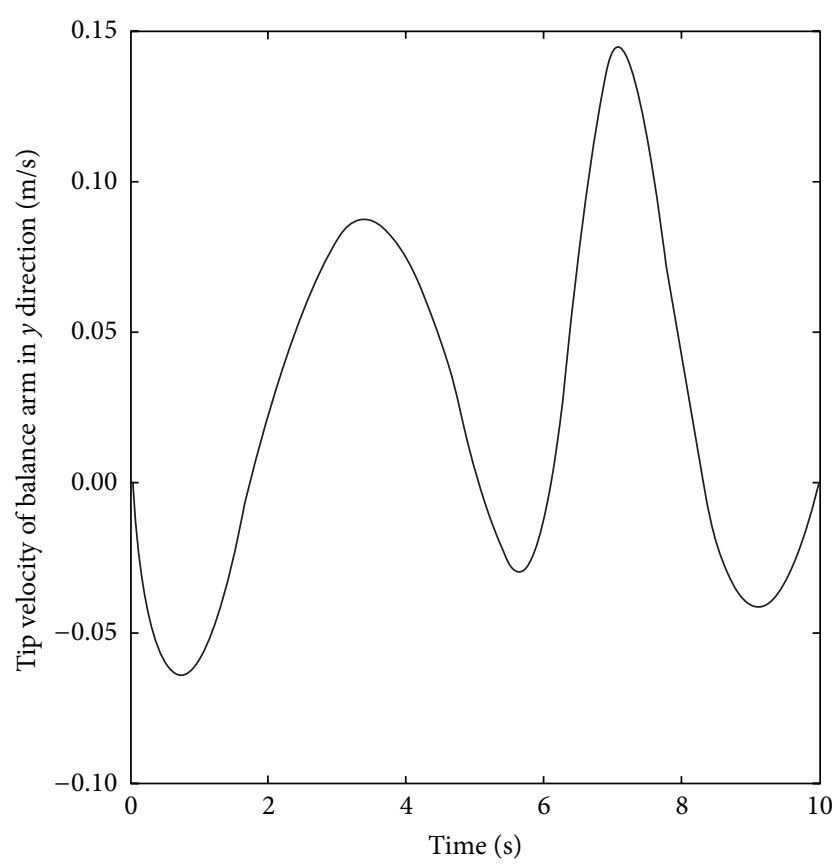

(b)

FiguRE 6: End effectors velocity of balance arm.

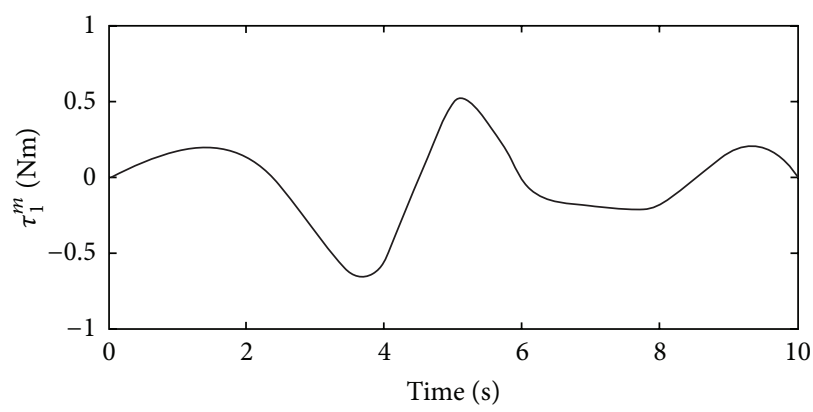

(a)

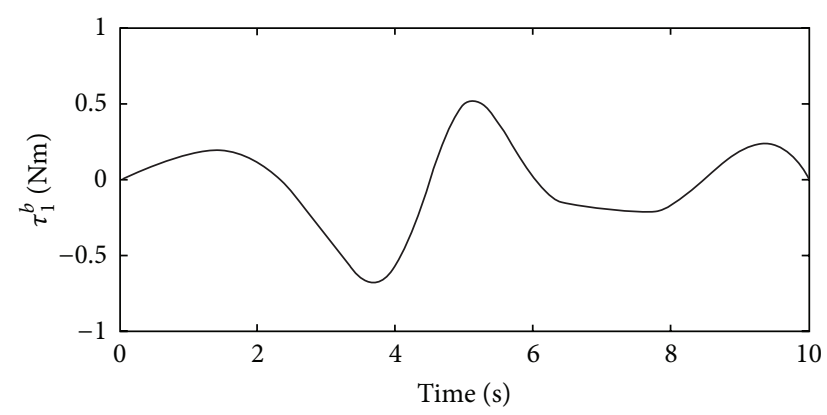

(c)

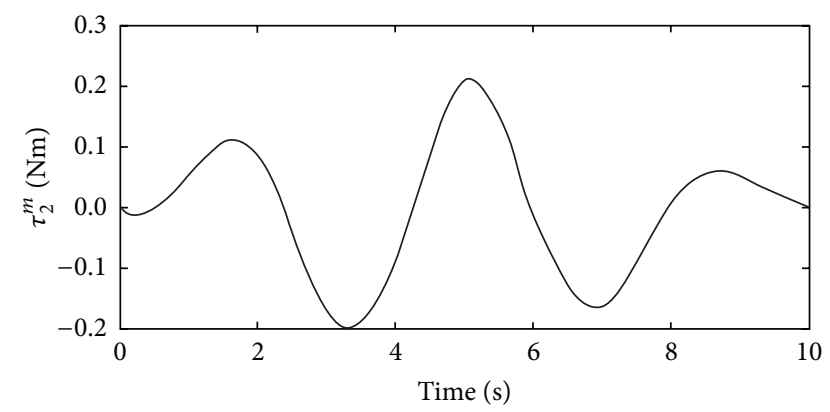

(b)

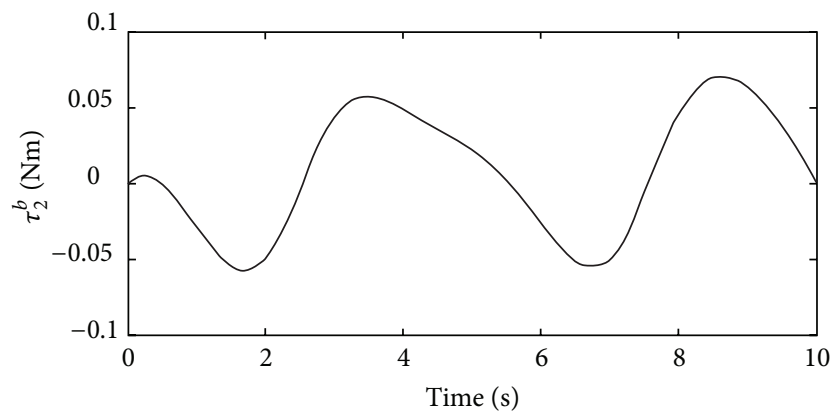

(d)

FIgURE 7: Manipulator torques of both the arms.

From the practical point of view, one should investigate the applicability of the method to more than two arms and threedimensional systems. One can also see the effect of increasing the degree of polynomial considering uncertainties in inertial parameters during path planning.

\section{Disclosure}

Part of this paper has been published in 15th National Conference on Machines and Mechanisms, IIT Madras, Chennai, 2011, India. 


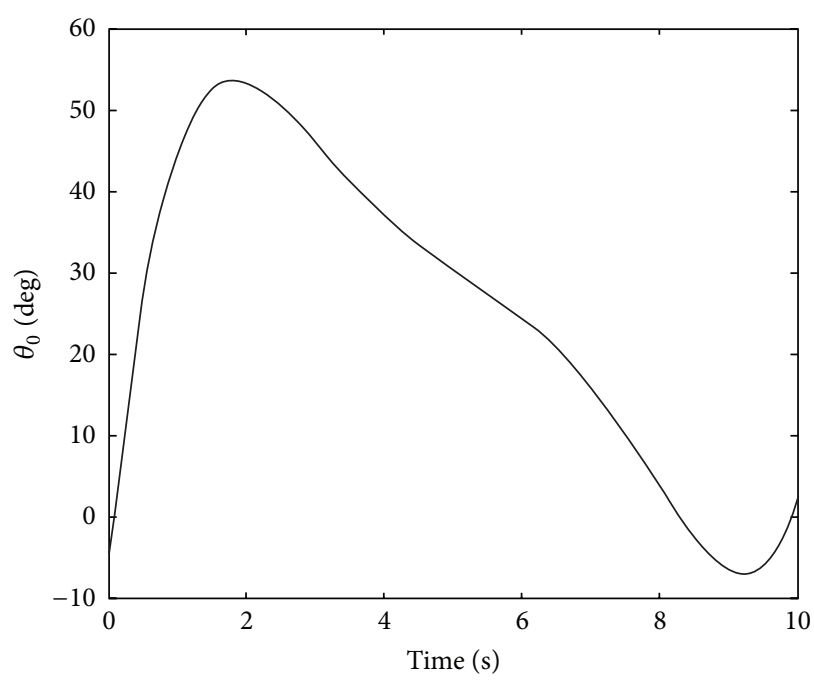

(a)

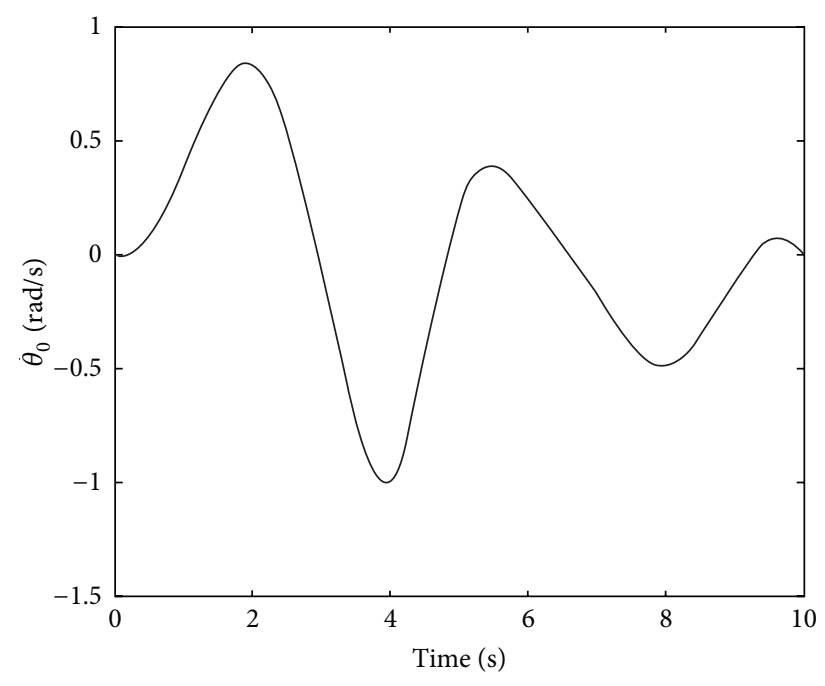

(b)

FIGURE 8: Base disturbance and base velocity variation with time.

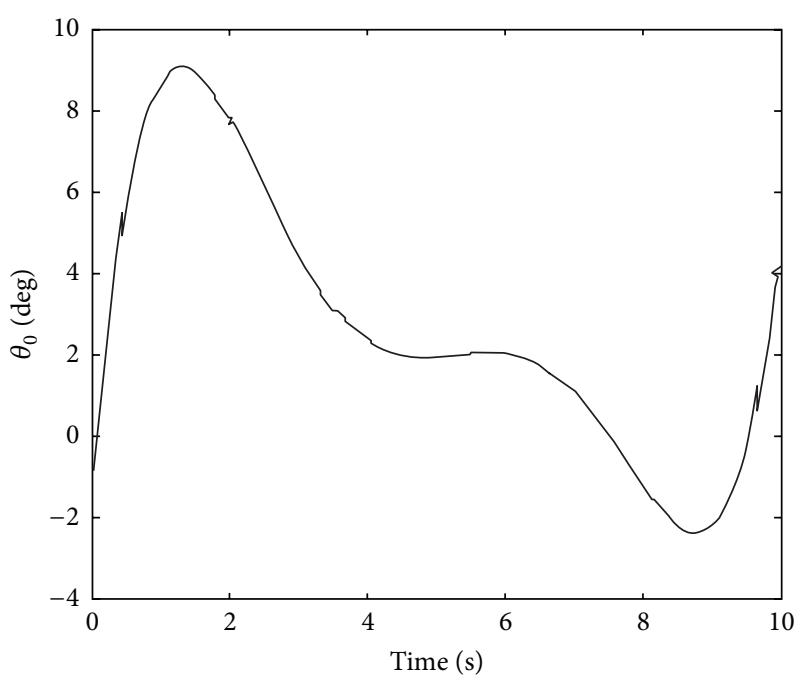

(a)

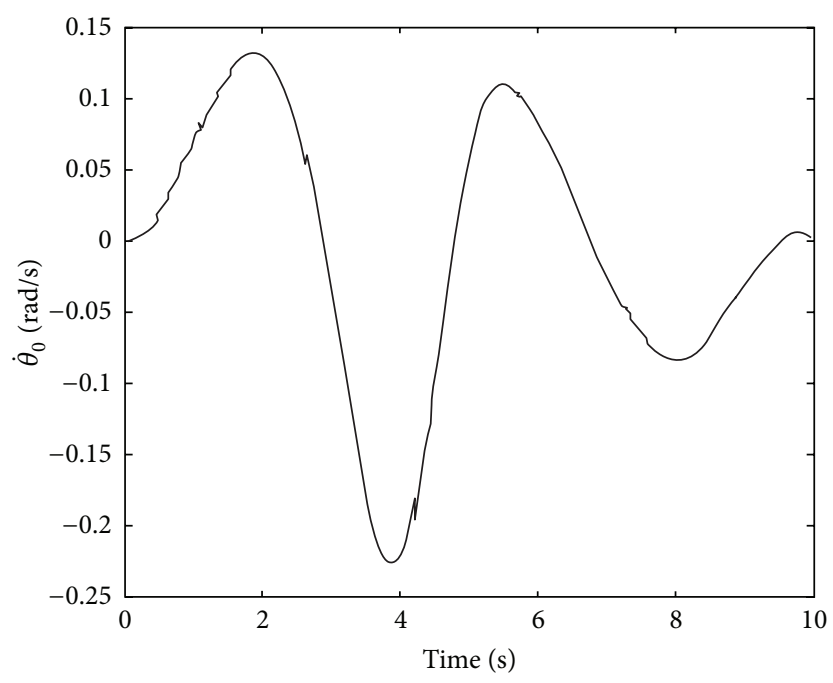

(b)

FIGURE 9: Base disturbance and base velocity variation with time for increased mass and inertia.

\section{Conflict of Interests}

The authors declare that there is no conflict of interests regarding the publication of this paper.

\section{References}

[1] Y. Nakamura and R. Mukherjee, "Nonholonomic path planning of space robots via a bidirectional approach," IEEE Transactions on Robotics and Automation, vol. 7, no. 4, pp. 500-514, 1991.

[2] K. Yoshida, R. Kurazume, and Y. Umetani, "Torque optimization control in space robots with a redundant arm," in Proceedings of the IEEE/RSJ International Workshop on Intelligent Robots and Systems (IROS '91), IEEE Cat. No. 91TH0375-6, pp. 16471652, Osaka, Japan, 1991.
[3] K. Yoshida, Space Robot Dynamics and Control: To Orbit, From Orbit, and Future, Department of Aeronautics and Space Engineering, Tohoku University Aoba 01, Sendai, Japan, 2000.

[4] E. Papadopoulos and S. A. A. Moosavian, "Dynamics and control of multi-arm space robots during chase and capture operations," in Proceedings of the IEEE/RSJ/GI International Conference on Intelligent Robots and Systems (IROS '94), vol. 3, pp. 1554-1561, Munich, Germany, September 1994.

[5] E. Papadopoulos, I. Tortopidis, and K. Nanos, "Smooth planning for free-floating space robots using polynomials," in Proceedings of the IEEE International Conference on Robotics and Automation, pp. 4272-4277, Barcelona, Spain, April 2005.

[6] L. Chen and Y. Guo, "Adaptive control of dual-arm space robot system in joint space," in Proceedings of the IEEE/RSJ International Conference on Intelligent Robots and Systems (IROS '06), pp. 5096-5099, Beijing, China, October 2006. 
[7] P. Huang, W. Xu, B. Liang, and Y. Xu, "Configuration control of space robots for impact minimization," in Proceedings of the IEEE International Conference on Robotics and Biomimetics (ROBIO '06), pp. 357-362, IEEE, Kunming, China, December 2006.

[8] S. Sagara and Y. Taira, "Cooperative manipulation of a floating object by some space robots: application of a tracking control method using the transpose of the generalized Jacobian matrix," in Proceedings of the 12th International Symposium on Artificial Life and Robotics, pp. 138-141, Oita, Japan, 2008.

[9] H. Zhao, C. Wang, and Z. Guo, "Coordinated dynamics control of a free-floating dual-arm space robot," Modern Applied Science, vol. 4, no. 5, pp. 175-182, 2010.

[10] B. Siciliano and O. Khatib, Springer Handbook of Robotics, Springer, Berlin, Germany, 2008.

[11] R. Merzouki, A. K. Samantaray, P. M. Pathak, and B. Ould Bouamama, Intelligent Mechatronic Systems: Modeling, Control and Diagnosis, Springer, London, UK, 2013.

[12] J. J. Craig, Introduction to Robotics, Mechanics and Control, Pearson Education, Upper Saddle River, NJ, USA, 2005.

[13] R. Rathi and P. M. Pathak, "Trajectory planning of dual arm free flying space robot using polynomial approach," in Proceedings of the National Conference on Machines and Mechanisms (NaCoMM'11), IIT, Chennai, India, November-December 2011.

[14] A. Ellery, An Introduction to Space Robotics, Praxis Publishing, Chichester, UK, 2000. 

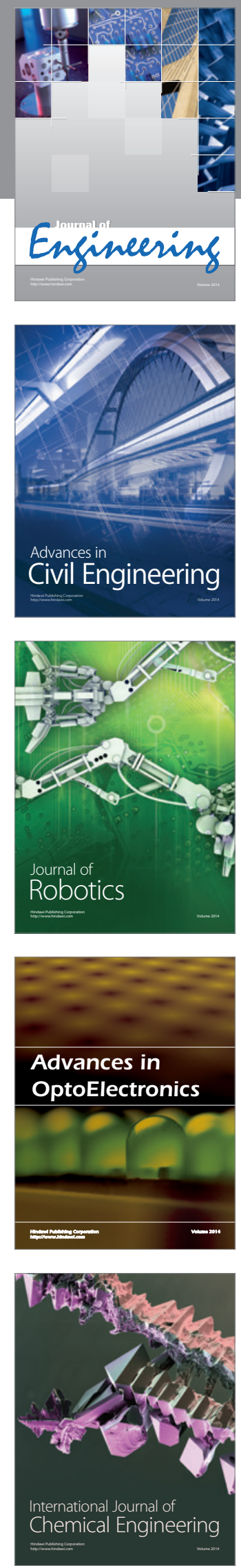

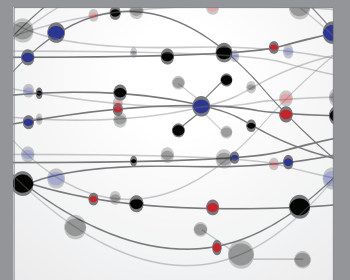

The Scientific World Journal
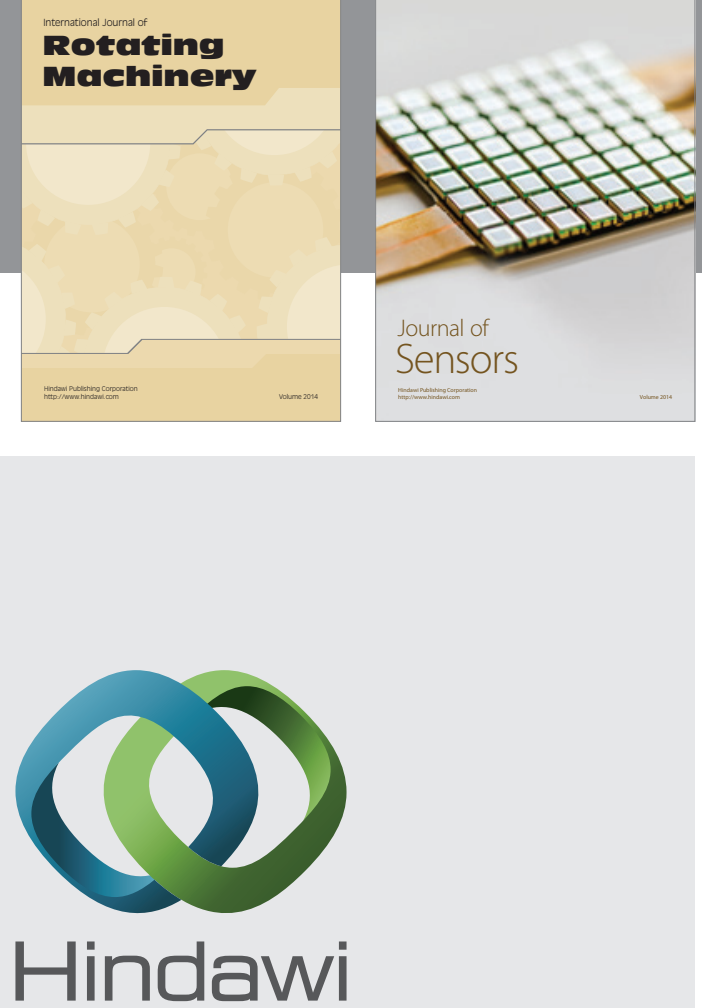

Submit your manuscripts at http://www.hindawi.com
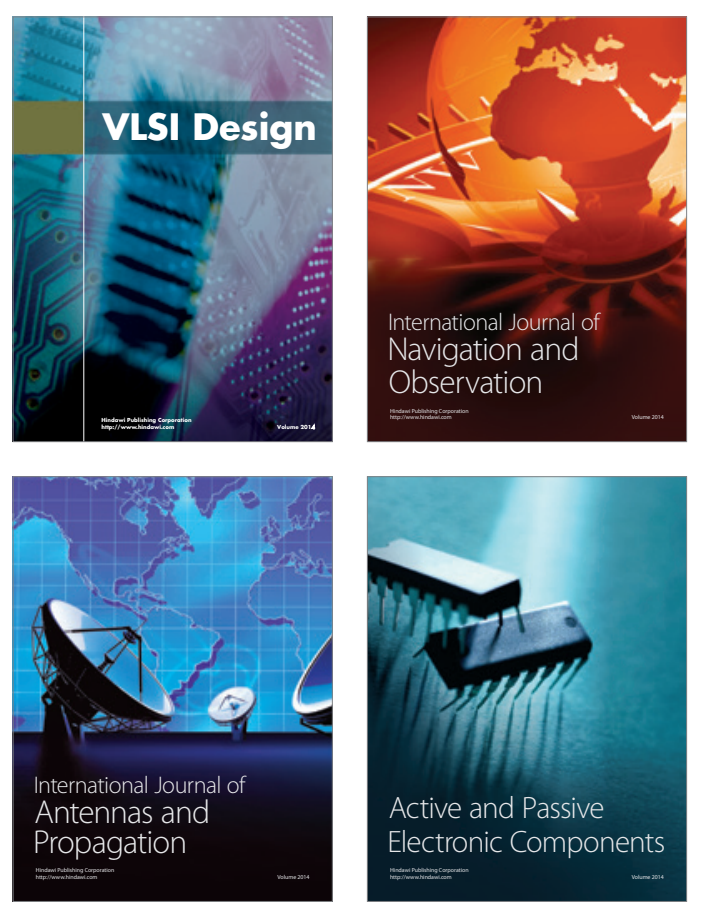
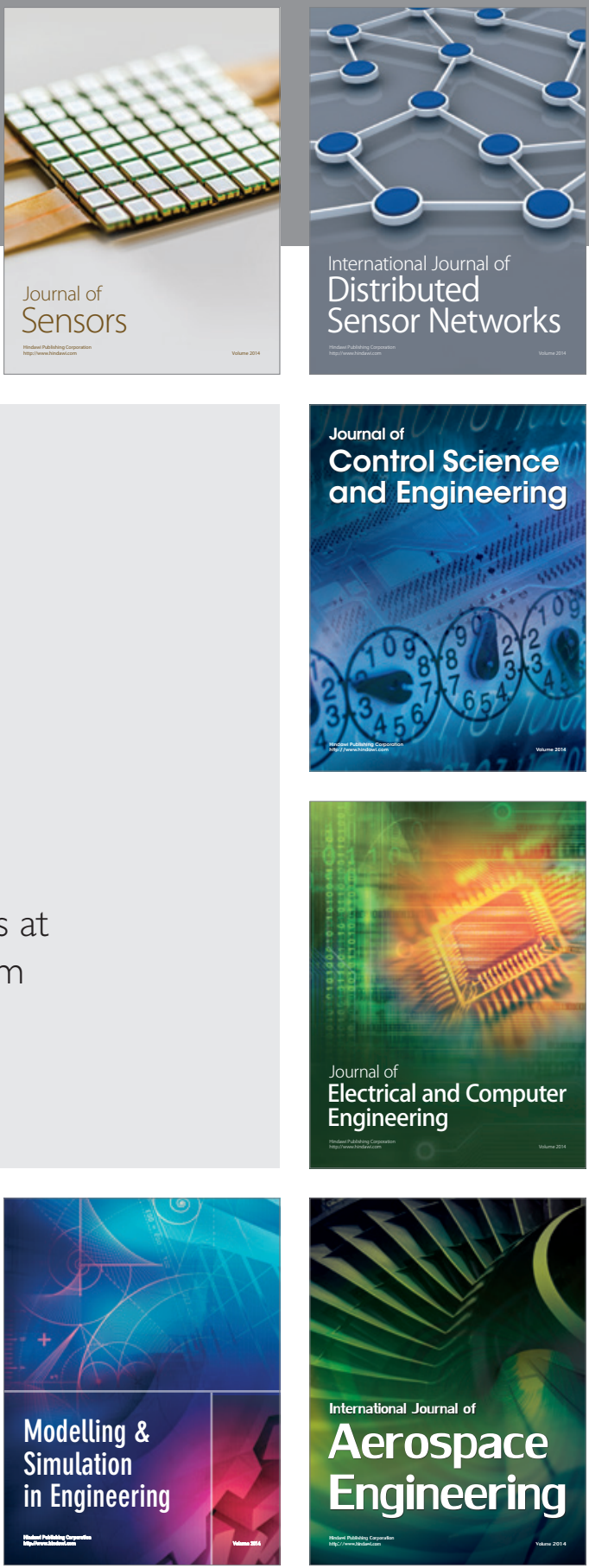

Journal of

Control Science

and Engineering
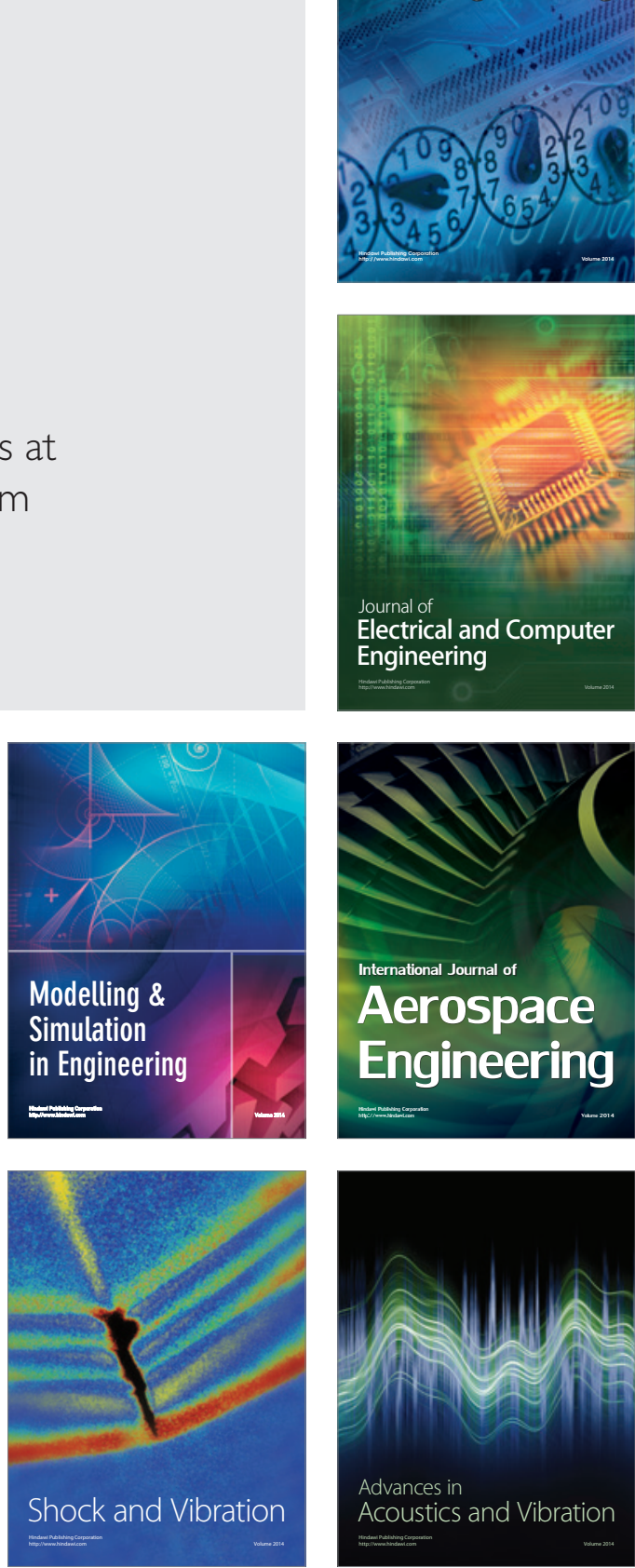